\section{Nuclear Magnetic Resonance Studies of Boron Trifluoride Complex Formation}

Hiar-resolution nuclear magnetic resonance spectroscopy affords a uniquely penetrating method of studying co-ordinate complex formation between boron trifluorido and electron donor substanees. The examples investigated so far comprise oxygen com. pounds (water, alcohols, ethers and ketones), but the method promises wide application. Details of a typical experiment follow. Fluorine magnetic resonance (at a frequency of $34.2 \mathrm{Mc} / \mathrm{s}$.) is observed in a quantitatively prepared liquid mixture of two donor substances (as, say, methanol and othanol) with boron trifluoride, the latter in quantity insufficient to saturate both donors. The temperature is controlled during a given measurement, the usable range extending from somewhat above room temperature to about $-100^{\circ} \mathrm{C}$. At sufficiently low temperatures two relatively narrow resonance peaks are seen, separated in the case cited by some $70 \mathrm{c} / \mathrm{s}$. These correspond to the co-ordination complexes of the respective donors. Measurement of the poak areas (assumed to be proportional to the volume concentrations) in mixtures of various composition indicates that reversible equilibrium is established between the two donors and their respective complexes. The mass law constant may thus be measured. The values at a series of different temperatures yield the corresponding enthalpy and standard entropy changes for the interconversion reaction.

Such an equilibrium constant is the ratio of tho mass law constants for the dissociation of the respective complexes into boron trifluoride and donor substance. A properly chosen series of studies with pairs of donors allows establishment of a 'boron trifluoride affinity' series; as an example, at a given temperature the substances water, methanol, ethanol, $n$-propanol and $n$-butanol constitute a regular saries, the water - boron trifluoride complex displaying the greatest stability. Mixtures containing three or more donor substances also demonstrate the order in such a series. Of particular interest is the comparison of isomeric substances, in which cases rather remarkable differences in boron trifluoride affinity are often observed.

As the temperature of such a mixture as that cited above is raised, the two fluorine resonance lines are observed to broaden and ultimately to merge. This behaviour is typical of chemical exchange effects, as already observed in previous studies by one of us (R. A. O. $)^{1}$.

In qualitative terms, the line broadening occurs as the pseudo-monomolecular rate constant for the interconversion approaches in value the frequency separa. tion of the lines. Systematic studies allow the evaluation of the rate constants at various temperatures, yielding the corresponding activation energies.

The equilibrium and kinetic studies indicated above are possible because of the rather striking variation of the fluorine resonance position ("chemical shift') as the donor substance is altered. The careful measurement of these chemical shifts, and their relation to structure and other properties of the co-ordination complexes, constitute another aspect of the entire programme. Of no less interest is the observation and interpretation of proton resonances. As an example, the formation of the boron trifluoride complex of ethanol results in a marked displacement (more than $100 \mathrm{c} . / \mathrm{s}$. as compared with pure ethanol) of the hydroxyl proton resonance toward lower magnetic field. The shift of the methylene resonance is in the same sense, but considerably smaller, while that of the methyl group resonance is negligible.

An extended programme of studies is in progress with the aim of following in systematic fashion the dependence of the various measurable quantities upon the molecular structure of the donor substances.

We wish to acknowledge the assistance of the Swiss National Fund, which has made possible the acquisition of the nuclear magnetic resonance spectrometer. We also appreciate the interest and advice of Prof. $P$. Huber, director of the Physical Institute.

This work was supported in part by a grant from the National Scioneo Foundation.

$$
\begin{aligned}
& \text { P. Drent } \\
& \text { R. A. OaG, Jư.* }
\end{aligned}
$$

Physical Institute, University of Basle, Switzerland. July 16.

* On leave from the Department of Chemistry, Stanford University, California.

1 Ogg, R. A., J. Chem. Phys., 22, 560 (1954); 26, 1341 (1957); Dise. Farad. Soc., 17, 215 (1954).

\section{Absorption Spectra of Flat Flames using a Multiple-Reflexion Technique}

MANY previous attempts to detect absorption bands of intermediate oxidation or pyrolysis products in premixed (Bunsen-type) flames have failed. There are several difficulties in studying absorption spoctra of flames; the reaction zone where intermediate products may be expected is usually extremely thin and often curved; it is necessary to have a background source the brightness of which exceeds that corresponding to the effective excitation temperature in the flame (which may sometimes be higher than the equilibrium flame temperature); the light boam may be thrown out of the flame by re. fraction effects ; and the concentration of the absorbing species may be low because of their instability or reactivity at high tomperatures, thus necessitating long path-length for their detection. To study a thin reaction zone, even if flat, it is necessary to restrict the light to a narrow beam of very small angular aperture.

We have recently made some progress in overcoming the difficulties by using (a) flat near-limit flames, of the type developed by Egerton and Powling ${ }^{1}$, having a reaction zone which may be a few millimetres thick and fairly flat, (b) a very bright xenon high-pressure lamp ('Osram' typ $X B O .162$ ) as background source, and $(c)$ a system of mirrors, of the type employed by White ${ }^{2}$, to reflect the beam of light backwards and forwards a number of times through the flame gases.

We have designed a metal flat-flame burner with a rectangular matrix, giving a flamo about $8 \mathrm{~cm}$. long. The burner has side-tubes containing the mirror system, with quartz windows for viewing. Front-surface aluminized mirrors were found to tarnish when exposed to some types of flame, and wo havo therefore used back-surface aluminized mirrors made from quartz, with the curvature of the surfaces so designed that images formed by reflexion from the front and back surfaces coincide.

Gradients of refractive index in the flame gases are low under our experimental conditions, and have not caused appreciable defocusing with less than twenty traverses. 